Cahiers $d u$ MONDE RUSSE

\section{Cahiers du monde russe}

Russie - Empire russe - Union soviétique et États indépendants

$46 / 4 \mid 2005$

L'invention d'une politique humanitaire

\title{
Sergej V. Kulikov, Bjurokratičeskaja elita Rossijskoj imperii
}

\section{Benjamin Guichard}

\section{(2) OpenEdition}

Journals

Édition électronique

URL : https://journals.openedition.org/monderusse/6570

DOI : 10.4000/monderusse. 6570

ISSN : $1777-5388$

\section{Éditeur}

Éditions de l'EHESS

\section{Édition imprimée}

Date de publication : 1 décembre 2005

Pagination : 869-871

ISBN : 2-7132-2057-2

ISSN : $1252-6576$

Référence électronique

Benjamin Guichard, "Sergej V. Kulikov, Bjurokratičeskaja elita Rossijskoj imperii », Cahiers du monde russe [En ligne], 46/4 | 2005, mis en ligne le 29 juin 2009, consulté le 03 septembre 2022. URL : http:// journals.openedition.org/monderusse/6570; DOI : https://doi.org/10.4000/monderusse.6570

Ce document a été généré automatiquement le 3 septembre 2022

Tous droits réservés 


\title{
Sergej V. Kulikov, Bjurokratičeskaja elita Rossijskoj imperii
}

\author{
Benjamin Guichard
}

\section{RÉFÉRENCE}

Sergej V. KULIKOV, Bjurokratičeskaja elita Rossijskoj imperii nakanune padenija starogo porjadka (1914-1917) [L'élite bureaucratique de l'empire russe à la veille de la chute de l'Ancien Régime (1914-1917)] Riazan, 2004, 472 p. (Novejšaja rossijskaja istorija : issledovanija i dokumenty, 4$)$

1 La Première Guerre mondiale en Russie a été le plus souvent analysée à travers le prisme des révolutions de 1917. Le délitement de l'appareil d'État, la société qui prend son destin en main pour l'organisation du ravitaillement et le secours des réfugiés, la sclérose d'un pouvoir refermé sur lui-même et les influences obscures de Rasputin scandent ces interprétations. La masse de la documentation mise en forme par l'historiographie ajoute parfois à la confusion et à la complexité du dossier : déposition de hauts fonctionnaires tsaristes devant les commissions d'enquête du Gouvernement Provisoire, abondance de mémoires des différents acteurs politiques de premier et de second plans publiés en émigration ou sous l'égide du jeune régime bolchevik, archives publiées dans les recueils et les revues soviétiques expliquent que l'étude du personnel politique de la fin du régime tsariste soit devenu un exercice fort prisé. Sur un sujet aussi controversé, le titre choisi par S.V. Kulikov fait preuve d'une grande modération, si ce n'est d'une grande réserve. On s'attendrait à un portrait de groupe des grands serviteurs des dernières années du tsarisme. L'introduction brosse un rapide tableau collectif qui permet de délimiter la notion d'élite bureaucratique en termes institutionnels. L'auteur s'est limité aux membres des quatre premières classes de la Table des rangs dont il ébauche le profil. Un précieux tableau statistique permet de préciser la composition de cette élite en termes de fortune et de revenus, d'origine sociale et d'âge. Mais les concessions aux sciences sociales s'arrêtent aux portes de l'ouvrage. S. V. Kulikov propose ici un examen 
détaillé et précis de la vie politique russe au cours de la Première Guerre mondiale. L'ouvrage est issu d'une thèse de doctorat soutenue en 1999.

La démonstration, organisée selon un plan chronologique, est consacrée au rapport de force entre deux lignes politiques définies par l'auteur comme celles du dualisme et du parlementarisme. La première regroupe les partisans d'une interprétation rigoriste des lois fondamentales de 1906 et considère que le partage du pouvoir législatif entre le tsar et les organes représentatifs doit être contrebalancé par une autonomie totale du pouvoir impérial à l'égard du parlement dans le domaine de l'exécution des lois et de l'exercice du pouvoir. À l'inverse, les parlementaristes sont partisans d'un contrôle du gouvernement par les organes représentatifs, confinant ainsi l'empereur à un rôle de chef de l'État aux pouvoirs limités non seulement par la loi, mais également par les compétences des autres institutions. L'auteur insiste sur le fait que ces deux lignes politiques ne doivent pas être comprises comme une opposition entre conservateurs et libéraux, mais comme deux interprétations d'un constitutionnalisme monarchique qui traverse l'ensemble de l'élite bureaucratique, qualifiée de réformiste et libérale. La position des constitutionnels-démocrates est ainsi réduite à une version radicale du parlementarisme. L'ouvrage suit la configuration des réseaux et des jeux d'alliance au sein des principaux lieux de pouvoir : le premier ministre, le gouvernement, ses relations avec le tsar, la Douma et les principales organisations sociales, le haut-commandement militaire et le Conseil d'État. Il met notamment en avant le poids et la profondeur de la crise politique de l'été 1915 et les enjeux de la constitution d'un "bloc progressiste ", c'est-à-dire la formation d'un gouvernement appuyé par les groupes de centre-gauche de la Douma. Le projet de constitution d'un poste extraordinaire de ministre de la Défense nationale en 1916 sous le gouvernement Sturmer est interprété comme une tentative de subordination de facto du pouvoir exécutif à la majorité parlementaire. Le dernier chapitre reconstitue les lignes de force à partir de la chute du gouvernement Sturmer, considérée comme une concession aux revendications des parlementaires et à ce titre comme une rupture essentielle. L'effondrement final du régime est attribué au revirement des grands-ducs qui choisissent de rompre avec la personne de l'empereur au profit des institutions parlementaires.

3 L'auteur met donc l'accent sur le fait que les racines de l'effondrement du régime sont à chercher dans les divisions du pouvoir. Il nuance ainsi fortement la vision qui explique la révolution par un divorce entre l'État et la société aggravé par la guerre. Son analyse des organisations sociales, Zemgor et Comité des industries de guerre, illustre bien ce schéma interprétatif. Les parlementaristes rassemblés autour du ministre de l'Agriculture Krivošejn et le haut commandement militaire protègent ces organisations qui offrent une base à leur politique en faveur de la constitution d'un bloc progressiste. L'ouvrage reconstitue avec précision l'évolution et l'éventail des subsides versés par l'État. Il cherche alors à montrer que ces organisations civiles, auxquelles l'État consacre une large part de ses ressources, sont les instruments de conflits de pouvoir. Les rumeurs liées à la germanophilie du premier ministre Sturmer à la fin de l'année 1916, par exemple,sont attribuées à une campagne orchestrée par ces associations à la suite d'arbitrages financiers en leur défaveur. Pour l'auteur, le développement de ces organismes n'est pas le fruit d'un phénomène endogène, mais le résultat d'une action délibérée et calculée de l'élite bureaucratique, destinée à faire basculer l'équilibre des forces au sein de l'appareil d'État au profit des partisans d'un système parlementaire. De même, la légende noire d'un Rasputin tenant l'empereur sous sa coupe et défaisant à 
l'envie les ministères avec la complicité de l'impératrice est battue en brèche par une analyse très fine des différents remaniements ministériels qui occupent une bonne part de l'ouvrage. La correspondance du tsar et de ses proches et la reconstitution de la chronologie des déplacements du moine permettent de minorer le poids du personnage dans les décisions de Nicolas II. À l'inverse, S. V. Kulikov souligne que les modifications de l'appareil gouvernemental s'expliquent par la recherche d'un compromis entre la nomination de ministres soutenus par la représentation nationale et le maintien d'un système dualiste. Ces deux exemples illustrent bien les limites de l'ouvrage. S'il faut saluer la richesse de l'appareil documentaire et la précision avec laquelle la chronique politique de la période est reconstituée, l'interprétation défendue manque parfois de nuances.

4 La place prépondérante accordée aux détails de la vie politique ne laisse qu'un rôle contingent au cours de la guerre et aux soubresauts de la société russe. Les organisations sociales, analysées uniquement à travers le spectre des luttes de pouvoir et des questions de financement, sont alternativement accusées d'âpreté au gain et de déloyauté à l'égard du régime qui les subventionne. Cependant leur rôle dans la prise en charge des réfugiés et la mobilisation économique du pays pourrait inviter à un autre éclairage. Le fait que le régime finance de façon aussi importante des organisations dont il connaît la défiance montre en effet l'importance des défaillances du système, fragilisé par les conséquences de l'engagement militaire du pays. De même, si la démonstration des fondements politiques du mythe des « forces sombres » du cercle Rasputin force l'adhésion, il semble difficile de conclure avec l'auteur que la figure de Nicolas II est celle d'un libéral centriste soucieux de compromis. L'attachement de l'empereur à une inspiration mystique que reconnaît S. V. Kulikov cadre mal en effet avec l'image d'homme politique rationnel et modéré qu'il défend, souvent à l'aide de syllogismes, contre une vision critique très intransigeante de l'opposition parlementaire. Pour l'essentiel, son argumentation repose sur une définition de la responsabilité politique ramenée à la fidélité à la personne du souverain. L'étude de la haute fonction publique, qu'il définit comme une élite de service, semblait pourtant exiger une interprétation du sens de l'État plus complexe. Le lecteur se perd parfois dans la combinaison très dense des sources, dont les témoignages sont tantôt récusés, tantôt utilisés pour appuyer des analyses iconoclastes sans qu'il soit toujours possible de démêler les considérations méthodologiques et critiques qui permettent à l'auteur d'asseoir ses interprétations. Ce sentiment semble partagé par le comité de rédaction de la collection. Celui-ci a choisi de faire figurer des réserves (p.205) à l'encontre des conclusions proposées dans le quatrième chapitre des deux dernières parties, consacré à la collusion entre l'État-Major et l'opposition - l'auteur attribue au chef de l'État-Major général, le général Alekseev, un projet d'enlèvement de la tsarine en octobre 1916 fomenté avec le prince L'vov en s'appuyant sur les mémoires de M. K. Lemke et S. P. Mel'gunov.

5 Si certaines interprétations de l'auteur et les termes de la typologie politique qu'il défend ne peuvent manquer de susciter des réserves, l'ouvrage de S. V. Kulikov offre une cartographie solide de la vie politique des dernières années du tsarisme. Au-delà de sa richesse, ce livre est une invitation convaincante à revisiter l'histoire politique des années de guerre. Il illustre ainsi avec force l'importance, la diversité et l'ampleur des réformes engagées dans tous les aspects de la vie politique entre l'été 1914 et la révolution de Février 1917. 